\title{
A Bacteriological Profile of Extended Spectrum B-Lactamase (ESBL) Producing Klebsiella from Pus \& Urine Samples From A Tertiary Care Medical College Hospital in Kerala.
}

\author{
Dr. Shreeram Astic Deshpande*, Dr. Divya MB, Dr. Chithira KG \\ Associate Professor, Department of Microbiology P.K. DAS Institute of Medical Sciences, Vaniamkulam, Kerala India
}

DOI: $10.36348 / \mathrm{sjpm} .2020 . \mathrm{v} 05 \mathrm{i} 09.003$

| Received: 03.09.2020 | Accepted: 13.09.2020 | Published: 24.09.2020

*Corresponding author: Dr. Shreeram Astic Deshpande

\section{Abstract}

Urinary tract, gastrointestinal, and pyogenic infections are the common hospital-acquired infections caused by members of Enterobacteriaceae. Among Enterobacteriaceae, Klebsiella and Escherichia coli has been the most commonly isolated species. Klebsiella are very well known to exhibit multidrug resistance. $\beta$-lactamase production is perhaps the single most important mechanism of resistance to penicillins and cephalosporins. E. coli \&Klebsiella possess a naturally occurring chromosomally mediated $\beta$-lactamase or plasmid mediated $\beta$-lactamases. These enzymes are thought to have evolved from penicillin binding proteins. This development was likely to be because of selective pressure exerted by $\beta$ lactam producing soil organisms found in the environment. In early 1960s, TEM-1 was the first plasmid mediated $\beta$ lactamase described in Gram-negative organisms. Another common plasmid mediated $\beta$-lactamase is SHV. Extended spectrum $\beta$-lactamases (ESBLs), enzymes that show increased hydrolysis of oxyimino- $\beta$-lactams, which include cefotaxime, ceftriaxone, ceftazidime, and aztreonam, have been reported increasingly in recent years. They belong to Ambler molecular class A and Bush-Jacoby functional group 2be. These enzymes have been identified in large numbers from different regions and are significantly detected in various E. coli \&Klebsiella strains. They have also been found in other members of Enterobacteriaceae such as Citrobacter spp, Enterobacter spp, Proteus spp and non-lactose fermenters like Pseudomonas aeruginosa. Today over 200 different ESBLs have been described. Major outbreak involving these resistant organisms has been reported all over the world in many members of the Enterobacteriaceae and Pseudomonas $s p p$, resulting in limitation of therapeutic options. Current knowledge of prevalence and incidence of ESBL production by commonly isolated organism such as E. coli and Klebsiella is necessary to understand the disease burden and to take necessary action to prevent the spread. Therefore the present study was conducted with an objective to find out the prevalence of ESBL producing Klebsiella and its antimicrobial resistance profile to formulate effective antibiotic strategy and plan a proper hospital infection control strategy to prevent the spread of these strains.

Keywords: CDL, Extended spectrum beta lactamase, Phenotypic, Klebsiella.

Copyright @ 2020: This is an open-access article distributed under the terms of the Creative Commons Attribution license which permits unrestricted use, distribution, and reproduction in any medium for non-commercial use (NonCommercial, or CC-BY-NC) provided the original author and sources are credited.

\section{INTRODUCTION}

Urinary tract, gastrointestinal, and pyogenic infections are the common hospital-acquired infections caused by members of Enterobacteriaceae. Among Enterobacteriaceae, Klebsiella and Escherichia coli has been the most commonly isolated species. Klebsiella are very well known to exhibit multidrug resistance. Extended antibiotic abuse, Length of stay in hospitals, severe illness, irrational use of third generation cephalosporin, and increased use of intravenous devices or catheters are important risk factors for infection with multidrug resistant Klebsiella [1]. ESBL producing strains are probably more prevalent than is currently recognized because they often remain undetected by routine susceptibility testing methods [6]. ESBL strains have been associated with resistance to other non $\beta$ lactam antibiotics like the aminoglycosides and chloramphenicol. Another property of these ESBL strains is that they might show a false sensitive zone of inhibition in the Kirby-Bauer disk diffusion method [7].

\section{MATERIALS AND METHODS}

Klebsiella isolates recovered from pus \& urine samples received in the bacteriology laboratory in the Department of Microbiology, P .K. Das Institute of Medical Sciences, Vaniamkulam, Kerala, from in and out patients. The Period of study was from February 2020 to July 2020. 


\section{Isolation and identification}

Urine samples collected in universal container, approximately $50 \mathrm{~mL}$ in amount were inoculated using an inoculating loop of $10 \mu \mathrm{L}$ volume calibration on cysteine lactose electrolyte deficient (CLED) agar plates. Pus Swabs were first rolled on blood agar plates and MacConkey agar plates and then streaked using an inoculating loop. All inoculated media were incubated aerobically overnight at $37^{\circ} \mathrm{C}$. On the basis of colony morphology, gram staining, motility, and biochemical reactions, the organisms were identified as Klebsiella. Biochemical reactions were performed by inoculating the colony in a nutrient broth at $37^{\circ} \mathrm{C}$ for $2-3$ hours. Following criteria was used for identification of Klebsiella [8].

Colony morphology: small 2-3 mm diameter, circular in shape, regular margin, flat, Mucoid, lactose fermenting, and translucent.

Gram staining: Gram-negative rods, $1-5 \times$ $0.3-0.5 \mu \mathrm{m}$ in size, uniformly stained with no particular arrangement, non-sporing, and capsulated. preparation.

Motility: non-motile bacteria in hanging drop

Biochemical reactions: Oxidase negative, catalase positive, $\mathrm{O} / \mathrm{F}$ test showed glucose fermentation, Non motile and abundant gas production, reduces nitrates to nitrites, indole negative, methyl red negative, Voges-Proskauer positive, citrate not utilized, lactose fermenter, triple sugar iron agar showed both butt and slant yellow with abundant gas production, lysine decarboxylase test positive.

\section{Antimicrobial susceptibility testing}

Antimicrobial susceptibility testing was done by Kirby-Bauer disk diffusion method as recommended by the Clinical Laboratory Standards Institute (CLSI) guidelines [9]. Commercially available antibiotic disks (HiMedia Labs, India) were used for antimicrobial susceptibility testing. The following antibiotic disks were used, ampicillin $(10 \mu \mathrm{g})$, piperacillin $(100 \mu \mathrm{g})$, piperacillin-tazobactam $\quad(100 / 10 \quad \mu \mathrm{g})$, amoxicillin/clavulanic acid $(20 / 10 \quad \mu \mathrm{g})$, cefoperazone/sulbactam $\quad(75 / 10 \quad \mu \mathrm{g})$, ceftazidime/clavulanate $(30 / 10 \mu \mathrm{g})$, cefoperazone $(75$ $\mu \mathrm{g})$, cefoxitin $(30 \mu \mathrm{g})$, ceftazidime $(30 \mu \mathrm{g})$, cefotaxime $(30 \mu \mathrm{g})$, ceftriaxone $(30 \mu \mathrm{g})$, cefepime $(30 \mu \mathrm{g})$, aztreonam $(30 \mu \mathrm{g})$, imipenem $(10 \mu \mathrm{g})$, amikacin $(30$ $\mu \mathrm{g})$, gentamicin $(10 \mu \mathrm{g})$, ciprofloxacin $(30 \mu \mathrm{g})$, ofloxacin $(5 \mu \mathrm{g})$, norfloxacin $(10 \mu \mathrm{g})$, and nitrofurantoin $(300$

$\mu \mathrm{g})$.

\section{Procedure}

Inoculum of $0.5 \mathrm{McFarland}$ standards turbidity was prepared in a nutrient broth from isolated colony of Klebsiella selected from 18-24 hour agar plates. Within
15 minutes, a sterile cotton swab was dipped into the inoculum suspension. The swab was rotated several times and pressed firmly against the inside wall of the tube above the fluid level and inoculated on the dried surface of a Mueller-Hinton agar (MHA) plate by streaking the swab over it. For even distribution of inoculum, the swab was streaked two more times at $60^{\circ}$ Cover the agar surface. After 3-5 minutes, antibiotic discs were applied and pressed down to ensure complete contact with agar surface.

The discs were distributed evenly to ensure a minimum distance of $24 \mathrm{~mm}$ from center to center. The plates are then inverted and incubated aerobically at $37^{\circ} \mathrm{C}$ within 15 minutes of disc application.

\section{Interpretation}

Diameter of zone of inhibitions were measured and recorded in millimeters with the help of sliding calipers and organism was labeled as sensitive, resistant, or intermediate as per CLSI 2012 guidelines [9].

\section{ESBL detection methods}

Klebsiella were first screened for ESBL production by phenotypic method and then phenotypic confirmatory test was done as per CLSI guidelines 2012 [9].

\section{(a) Phenotypic screening of ESBL}

CLSI 2012 has recommended the use of any of the following antibiotic discs for screening of ESBL producers. Antibiotic disks of ceftazidime, aztreonam, cefotaxime, and ceftriaxone were used. More than one of these agents was used for screening to improve the sensitivity of ESBL detection, as CLSI has recommended the method only in 2012 guidelines.

\section{i) Procedure}

Inoculum with turbidity equivalent to 0.5 McFarland standards was prepared from colonies on agar plates. MHA plates were inoculated by lawn culture method using a sterile cotton swab. With a sterile forceps ceftazidime, cefotaxime, ceftriaxone, and aztreonam disks were placed on the MHA plate and the plate was incubated at $35^{\circ} \mathrm{C}$ for $18-24$ hours.

\section{ii) Interpretation of results}

Zones given below, against respective antibiotic indicate potential ESBL producer. If any strain was suspected as ESBL producer then phenotypic confirmatory tests were done.

1 Ceftazidime $\leq 22 \mathrm{~mm}$

2 Aztreonam $\leq 27 \mathrm{~mm}$

3 Ceftriaxone $\leq 25 \mathrm{~mm}$

4 Cefotaxime $\leq 27 \mathrm{~mm}$

\section{(b) Phenotypic confirmatory methods} Confirmatory test was done by two methods. 


\section{i) Double disk diffusion test}

Double disk approximation or double disk synergy (DDS) is a disk diffusion test in which $30 \mu \mathrm{g}$ antibiotic disks of ceftazidime, ceftriaxone, cefotaxime, and aztreonam are placed on the lawn culture plate of $E$. coli on MHA, $30 \mathrm{~mm}$ (center to center) from the amoxicillin/clavulanic acid $(20 / 10 \mu \mathrm{g})$ disk. This plate was incubated aerobically overnight at $37^{\circ} \mathrm{C}$ and examined for an extension of the edge of zone of inhibition of antibiotic disks toward the disk containing clavulanate. It is interpreted as synergy, indicating the presence of an ESBL.

\section{ii) Cephalosporin/clavulanate combination disks}

Cefotaxime $(30 \mu \mathrm{g})$ or ceftazidime disks with $(30 \mu \mathrm{g})$ or without clavulanate $(10 \mu \mathrm{g})$ was used for phenotypic confirmation of the presence of ESBL as recommended by CLSI 2012 guidelines [9]. A lawn culture of $E$. coli was made on the MHA plate and disks were placed at an appropriate distance from each other and incubated aerobically overnight at $37^{\circ} \mathrm{C}$. A difference in zone of inhibition of $\geq 5 \mathrm{~mm}$ of either of cephalosporin disks and their clavulanate containing disks indicates production of ESBL.

\section{STATISTICAL ANALYSIS}

Chi-square test is used for statistical analysis of the data. A ' $P$ value' less than 0.05 were considered statistically significant.

\section{RESULTS}

Among all the isolates, only 185 non-duplicate isolates of Klebsiella that showed resistance to third generation cephalosporins were included in this study without applying any selection criteria for the patients. Klebsiella was isolated in the highest number from urine followed by pus samples.

Among 185 isolates, 95 isolates were obtained from inpatients samples and 90 isolates were isolated from outpatients In our study, it is observed that Klebsiella is $100 \%$ susceptible to imipenem and meropenem. Susceptibility to third generation cephalosporin is between 40 and $65 \%$, which is quite low. Susceptibility to piperacillin/tazobactam, amoxicillin/clavulanic acid, cefoperazone/sulbactam, ceftazidime/clavulanate, amikacin, norfloxacin (only in urine), and ciprofloxacin was good and between 50 and $90 \%$. There was $100 \%$ resistance to ampicillin of the total Klebsiella isolates, $125(67.55 \%)$ isolates were ESBL producers and $60(32.4 \%)$ isolates were nonESBL producers. Among ESBL producers, the maximum number was isolated from urine $(54.67 \%)$ followed by pus $(45.33 \%)$ of the 95 organisms isolated from 95 in-patients, 64 (67.3\%) were ESBL producers while $31(34.4 \%)$ out of 90 from out-patients were ESBL producers. ESBL producers were more common among inpatients than out-patients. ESBL and nonESBL producers compared among in- and out-patients give significant result $(P<0.001)$. Antimicrobial sensitivity pattern of ESBL producing Klebsiella from urine and pus showed that it was $100 \%$ susceptible to imipenem and meropenem.

\section{DISCUSSION}

The discovery and development of antibiotics was undoubtedly one of the greatest advances of modern medicine. Unfortunately the emergence of antibiotic resistance bacteria is threatening the effectiveness of many antimicrobial agents. This has increased the hospital stay of the patients, which in turn causes economic burden. In this study overall incidence of ESBL producing Klebsiella was studied based on laboratory findings and includes the patients attending the outpatient and in-patient departments of P .K. Das Institute of Medical Sciences, Vaniamkulam, Kerala .On screening with third generation cephalosporin, a total of 185 Klebsiella cisolates were selected and studied for their antimicrobial susceptibility and ESBL production. In this study, samples were collected from IP/OPDs. All the 185 isolates of Klebsiella were tested by Kirby-Bauer disk diffusion method for antimicrobial susceptibility pattern.

Highest susceptibility was found to imipenem \&meropenem $\quad(100 \%) \quad$ followed by piperacillin/tazobactam (87.22\%), cefoperazone/sulbactam (76.67\%), amoxicillin/clavulanic acid $(75.55 \%)$, and ceftazidime/clavulanate $(66.11 \%)$. Klebsiella were resistant to most of the drugs used as first line drugs. A low susceptibility was observed with third generation cephalosporin (cefotaxime, ceftazidime, and ceftriaxone) (31.11 and $35.55,38.33 \%$, respectively), cephamycin (cefoxitin) (31.11\%), monobactam (aztreonam) (31.11\%), piperacillin (33.33\%), cefoperazone $(27.77 \%)$, and cefepime $(35.55 \%)$.

Akram et al. and Padmini et al also reported $100 \%$ susceptibility of urinary isolates of Klebsiella to imipenem $[11,11]$. Menon et al. in their study reported almost similar results of susceptibility for imipenem, piperacillin/tazobactam, cefoperazone/sulbactam, and ceftazidime/clavulanate with slight variation [12]. Similar susceptibility patterns were also observed in studies conducted outside India. Kibret et al. showed a high resistance to amoxicillin $(86.0 \%)$ and tetracycline $(72.6 \%)$ but a significantly high degree of susceptibility to nitrofurantoin $(96.4 \%)$, norfloxacin $(90.6 \%)$, and gentamicin (79.6\%) [13]. Bamford et al. demonstrated a significant decline in susceptibility to $\beta$ lactam antibiotics and fluoroquinolones, while susceptibility to amikacin and gentamicin remained significantly high [14].

In the present study, out of 185 Klebsiella, $67.55 \%$ were ESBL producers by phenotypic confirmatory methods. The incidence of ESBL producing Klebsiella varies from country to country and from center to center. In the United States, ESBL 
producing Klebsiella ranges from 0 to $25 \%$ with the average being around 3\%. [15]. In Japan, the prevalence of ESBL producing Klebsiella is $<0.1 \%$ [16]. In Asia, the percentage of ESBL production is $4.8,8.5$, and up to $12 \%$ in Korea, Taiwan, and Hong Kong, respectively $[17,19]$. In India, the percentage of ESBL producers ranges from 22 to $75 \%$ [20, 23].

In our study, incidence of ESBL among inpatients and outpatients was $67.3 \%$ and $34.4 \%$, respectively. Although the incidence of ESBL in outpatients is less than in-patients, it is common in communities. This is because ESBL producing Klebsiella isolates were wide spread among both inpatients and out-patients. This observation therefore confirms the assertion by Pitout et al that ESBL producers are indeed as much a problem in the communities as in the hospitals [24].

ESBL producers may have spread through communities, especially those with poor hygienic and sanitation conditions, through fecal contamination of soil and water, since most patients with ESBL producers may have had their gastrointestinal tracts colonized for a long period of time by these organisms as was reported by Paterson and Bonomo (2005) [5]. In vitro susceptibility studies of ESBL producing Klebsiella isolated from pusand urine showed that drug resistance was higher in ESBL producers than nonESBL producers. Analysis of antimicrobial susceptibility pattern of ESBL producing Klebsiella isolates demonstrated high susceptibility rates to imipenem \& meropenem (100\%), $\beta$ lactam/ $\beta$-lactamase inhibitor combination drugs such as

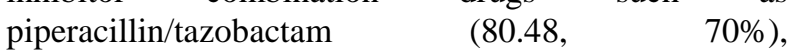
cefoperazone/sulbactam $\quad(70.73, \quad 80 \%)$, ceftazidime/clavulanate $\quad(70.73, \quad 70 \%)$, amoxicillin/clavulanic acid (68.29, 70\%), and aminoglycosides such as amikacin $(73.17,60 \%)$ and gentamicin $(68.29,60 \%)$ from urine and blood, respectively.

High resistance rates were observed to penicillins such as ampicillin and piperacillin, third and fourth generation cephalosporins and fluoroquinolones. Norfloxacin and nitrofurantoin have good susceptibility against ESBL producing $E$. coli isolated from urine. So these drugs are recommended for the treatment of infections caused by ESBL producing E. coli. The carbapenems, on the other hand, should be used to treat only serious or life threatening infections in order to minimize cases of carbapenem resistance, though rare [5]. In a study conducted by Ankur et al on clinical isolates of ESBL producing E. coli, resistance found to amikacin was $14.7 \%$, gentamicin $66.7 \%$, trimethoprim/sulfamethoxazole $79.1 \%, \quad$ and ciprofloxacin $93.8 \%$ [25]. Maina et al documented a higher proportion of isolates resistant to ciprofloxacin, levofloxacin, and tetracycline, and approximately $100 \%$ sensitivity to carbapenems [26]. Al-Zarouni et al. also demonstrated high resistance rates to fluoroquinolones and cephalosporins and higher susceptibility rates to carbapenems and amikacin [27].

The ESBL producing Klebsiella are a cause of concern to the microbiologist as well as to the clinicians, particularly the multi drug resistant strains. Correct choice of antimicrobial agents according to the sensitivity profile is essential for appropriate empirical treatment. In the present study, no resistance was shown to carbapenems (imipenem \& meropenem). So, we suggest the use of carbapenems as the drug of choice for ESBL producers causing life threatening infections. However, antimicrobial susceptibility testing should be performed for each strain before prescribing antibiotics. The carbapenem should be used as a reserve drug only in cases of multi drug resistant strains. Carbapenem resistance in Klebsiella is only beginning to emerge as a clinical issue, yet the attention it has already received serves to underscore the seriousness of the problem.

\section{REFERENCES}

1. Chaudhary, U., Aggarwal, R. (2004). Extended spectrum $\beta$-lactamases (ESBL)-an emerging threat to clinical therapeutics. Indian J Med Microbiol, 22(2):75-80.

2. Bradford, P.A. (2001). Extended spectrum $\beta$ lactamases in the $21^{\text {st }}$ century: characterization, epidemiology and detection of this important threat. Clin Microbiol Rev, 14:933- 951.

3. Rodrigues, C., Joshi, P., Jani, S.H., Alphonse, M., Radhakrishnan, R., Mehta, A. (2004). Detection of $\beta$-lactamases in nosocomial gram negative clinical isolates. Indian J Med Microbiol, 22(4):247-250.

4. Bush, K., Jacoby, G.A. (2010). Updated functional classification of beta-lactamases. Antimicrob Agents Chemother, 54(3):969-976.

5. Paterson, D.L., Bonomo, R.A. (2005). Extendedspectrum $\beta$-lactamases: a clinical update. Clin Microbiol Rev, 18:657-686.

6. Subha, A., Ananthan, S. (2002). Extended spectrum $\beta$-lactamase (ESBL) mediated resistance to third generation cephalosporins among Klebsiella pneumoniae in Chennai. Indian J Med Microbiol, 20(2):92-95.

7. Khurana, S., Taneja, N., Sharma, M. (2002). Extended Spectrum $\beta$ lactamase mediated resistance in urinary tract isolates of family Enterobacteriaceae. Ind J Med Res, 116:145-9.

8. Pamela, B.C. (2007). Enterobacteriaceae: Escherichia, Klebsiella, Proteus and other genera. In: Collee JG, Marmion BP, Fraser AG, Simmons A, editors. Mackie and McCartney Practical Medical Microbiology. 14th ed. UK: Churchill Livingstone, 361-367.

9. Chaudhary, U., \& Aggarwal, R. (2004). Extended spectrum-lactamases (ESBL)-An emerging threat to clinical therapeutics. Indian journal of medical microbiology, 22(2), 75. 
10. Akram, M., Shahid, M., Khan, A.U. (2007). Etiology and antibiotic resistance patterns of community-acquired urinary tract infections in the JNMC Hospital Aligarh, India. Ann Clin Microbiol Antimicrob, 6:4.

11. Padmini, B.S., Appalaraju, B. (2004). Extended spectrum $\beta$ lactamases in urinary isolates of $E$. coli and $K$. pneumoniae - prevalence and susceptibility pattern in a tertiary care hospital. Indian $\mathrm{J} \mathrm{Med}$ Microbiol, 22(3):172-4.

12. Menon, T., Bindu, D., Kumar, C.P., Nalini, S., Thirunarayan, M.A. (2006). Comparison of double disc and three dimensional methods to screen for ESBL producers in a tertiary care hospital. Indian J Med Microbiol, 24(2):117-120.

13. Kibret, M., Abera, B. (2011). Antimicrobial susceptibility of $E$. coli from clinical sources in northeast Ethiopia. Afr Health Sci, 11(1):S40-S45.

14. Bamford, C., Bonorchis, K., Ryan, A. (2012). Antimicrobial susceptibility patterns of Escherichia coli strains isolated from urine samples in South Africa from 2007- 2011. South Afr J Epidemiol Infect, 27(2):46-52.

15. National Nosocomial Infections Surveillance System. (2004). National Nosocomial Infections Surveillance (NNIS) system report, data summary from January 1992 through June 2004, issued October 2004. Am J infect control, 32, 470-485.

16. Yagi, T., Kurokawa, H., Shibata, N., Shibayama, K., \& Arakawa, Y. (2000). A preliminary survey of extended-spectrum $\beta$-lactamases (ESBLs) in clinical isolates of Klebsiella pneumoniae and Escherichia coli in Japan. FEMS microbiology letters, 184(1), 53-56.

17. Pai, H., Lyu, S., Lee, J. H., Kim, J., Kwon, Y., Kim, J. W., \& Choe, K. W. (1999). Survey of extended-spectrum $\beta$-lactamases in clinical isolates of Escherichia coli and Klebsiella pneumoniae: prevalence of TEM-52 in Korea. Journal of Clinical Microbiology, 37(6), 1758-1763.

18. Yan, J. J., Wu, S. M., Tsai, S. H., Wu, J. J., \& Su, I. J. (2000). Prevalence of SHV-12 among Clinical Isolates Ofklebsiella pneumoniae Producing Extended-Spectrum $\quad \beta$-Lactamases and Identification of a Novel AmpC Enzyme (CMY-8) in Southern Taiwan. Antimicrobial Agents and Chemotherapy, 44(6), 1438-1442.

19. Ho, P. L., Tsang, D. N. C., Que, T. L., Ho, M., \& Yuen, K. Y. (2000). Comparison of screening methods for detection of extended- spectrum $\beta$ lactamases and their prevalence among Escherichia coli and Klebsiella species in Hong Kong. Apmis, 108(3), 237-240.

20. Agrawal, P., Ghosh, A. N., Kumar, S., Basu, B., \& Kapila, K. (2008). Prevalence of extendedspectrum $\beta$-lactamases among Escherichia coli and Klebsiella pneumoniae isolates in a tertiary care hospital. Indian Journal of Pathology and Microbiology, 51(1), 139.

21. Aruna, K., \& Mobashshera, T. (2012). Prevalence of extended spectrum beta-lactamase production among uropathogens in South Mumbai and its antibiogram pattern. EXCLI journal, 11, 363.

22. Dalela, G. (2012). Prevalence of extended spectrum beta lactamase (ESBL) producers among gram negative bacilli from various clinical isolates in a tertiary care hospital at Jhalawar, Rajasthan, India. Journal of Clinical and Diagnostic research, 6(2), 182-187.

23. Abhilash, K. P., Veeraraghavan, B., \& Abraham, O. C. (2010). Epidemiology and outcome of bacteremia caused by extended spectrum betalactamase (ESBL)-producing Escherichia coli and Klebsiella spp. in a tertiary care teaching hospital in south India. $J$ Assoc Physicians India, 58(Suppl), 13-17.

24. Pitout, J. D. D., Reisbig, M. D., Venter, E. C., Church, D. L., \& Hanson, N. D. (2003). Modification of the double-disk test for detection of Enterobacteriaceae producing extendedspectrum and AmpC $\beta$-lactamases. Journal of Clinical Microbiology, 41(8), 3933-3935.

25. Ankur, G., Prasad, K. N., Amit, P., Sapna, G., Ujjula, G., \& Archana, A. (2009). Extended spectrum $\beta$-lactamases in Escherichia coli \& Klebsiella pneumoniae \& associated risk factors. Indian Journal of Medical Research, 129(6), 695-700.

26. Maina, D., Makau, P., Nyerere, A., \& Revathi, G. (2013). Antimicrobial resistance patterns in extended-spectrum $\quad \beta$-lactamase producing Escherichia coli and Klebsiella pneumoniae isolates in a private tertiary hospital, Kenya. Microbiology Discovery, 1(5), 1.

27. Al-Zarouni, M., Senok, A., Rashid, F., Al-Jesmi, S. M., \& Panigrahi, D. (2008). Prevalence and antimicrobial susceptibility pattern of extendedspectrum beta-lactamase-producing Enterobacteriaceae in the United Arab Emirates. Medical Principles and Practice, 17(1), 32-36. 